\title{
Giant Frog of the Floor of the Mouth
}

\author{
Belhaj $\mathrm{N}^{1,3^{*}}$, Nekro $\mathrm{C}^{1}$, Rahim $\mathrm{H}^{1}$, Boumendil I ${ }^{1}$, Bencheikh $\mathrm{R}^{2}$, Benbouzid $\mathrm{MA}^{2}$, Essakalli L ${ }^{2}$
}

${ }^{1}$ Resident physician in otorhinolaryngology, Department of Otorhinolaryngology, Head and Neck Surgery, Ibn Sina University Hospital, Rabat, Morocco

${ }^{2}$ Professor of otorhinolaryngology, Department of Otorhinolaryngology, Head and Neck Surgery, Ibn Sina University Hospital, Rabat, Morocco

${ }^{3}$ Faculty of Medicine and Pharmacy of Rabat, Mohammed V University, Rabat, Morocco

DOI: $10.36347 /$ sjmcr.2020.v08i04.004

| Received: 26.03.2020 | Accepted: 02.04.2020 | Published: 05.04.2020

*Corresponding author: Belhaj Najoua

Abstract

The sublingual frog or ranula is a sialocele related to mucosal cystic retention secondary to obstruction of the sublingual gland or its duct (more rarely accessory glands). We distinguish the simple and plunging shape. The simple form is limited to the sublingual gland and corresponds to a true epithelial cyst. The anatomical location is specified by imagery: it is located in the floor of the mouth above the mylohyoid muscle and inside the genio-hyoid muscle. The plunging shape corresponds to an extension in the submandibular space by the free edge of the mylohyoid muscle. Most often we visualize an extension of the cyst in the submandibular region, this is what we report in this work which reports the case of a patient who consulted for mass under mandibular and whose diagnosis is posed by imaging, biology and confirmed after surgical exercise by anatompathology.

Keywords: Giant, sublingual frog, Floor, Mouth, anatompathology.

Copyright @ 2020: This is an open-access article distributed under the terms of the Creative Commons Attribution license which permits unrestricted use, distribution, and reproduction in any medium for non-commercial use (NonCommercial, or CC-BY-NC) provided the original author and source are credited.

\section{INTRODUCTION}

A Frog corresponds to an extra-glandular and extra-ductal collection of saliva in the floor of the mouth from a sublingual gland. More rarely it can come from an injury to the canal of a submandibular gland.

Treatment for frogs is controversial and includes marsupialization, excision of the sublingual gland, excision of the frog $+/$ - excision of the sublingual gland, and sclerotherapy.

Removal of the sublingual gland is the key to reducing recurrence. We report in this work the observation of a patient who consulted for a swelling under left angular mandibular evolving for 10 years.

\section{Case Report}

It is Z.O patient seen in ENT consultation for swelling above the hyoid evolving for 10 years gradually increasing in size the clinical examination finds a sub mental mass with an intraoral component which occupies an important place of the buccal floor. in intraoral swelling is $6 \mathrm{~cm}$ in diameter; in the submental region, it is a well-defined mass, rounded, soft, 4 $\mathrm{cm}$ in diameter, mobile in both planes, Bi digital finger palpation allows to specify that it is a single mass, with intraoral and cervical expression.

The rest of the cervico-facial examination is normal. The injected CT scan as well as the ultrasound showed a benign-looking cystic mass of the floor of the mouth with liquid contents directed more towards a giant frog (figure 1.2).

A fine needle mass puncture found saliva amylase dosage was positive. The patient benefited from a removal of this bulky cyst under general anesthesia by mixed, intraoral and cervical routes, because an impossibility of removing the cyst by intraoral route considering its volume (Figure 3.4). 


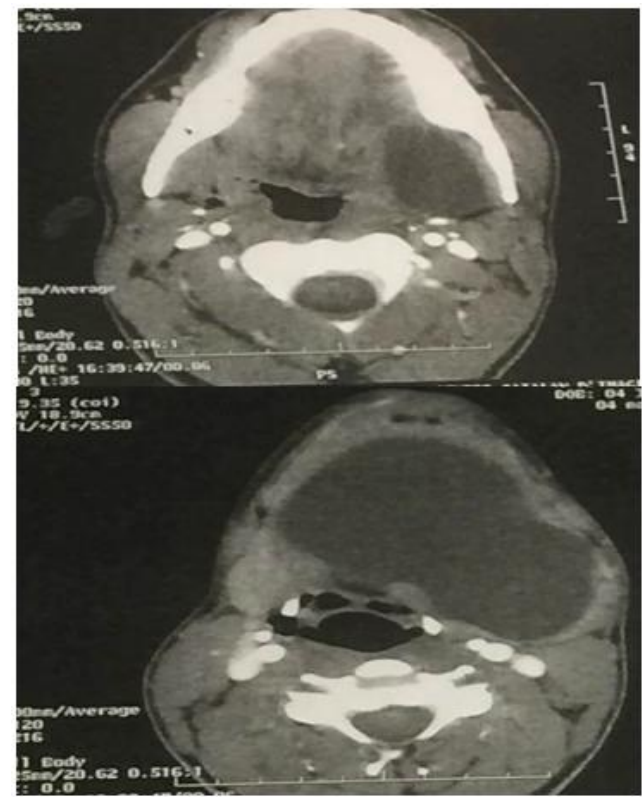

Fig-1.2: axial CT sections showing a mass of liquid contents of the floor of the mouth

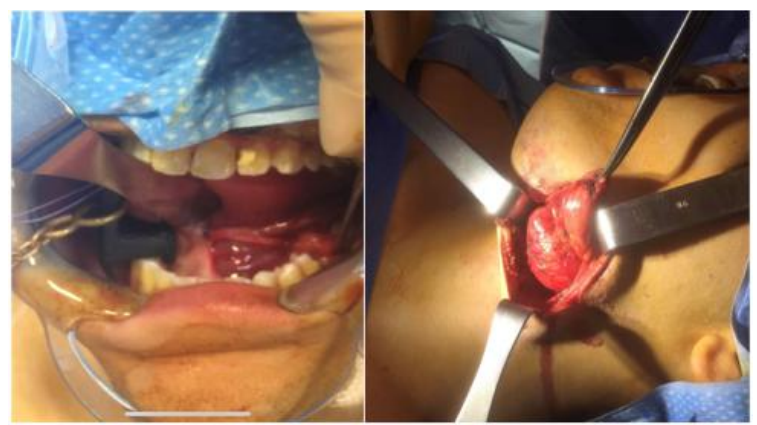

Fig-3.4: Intraoperative images of the frog exercise gesture given the size impossible by intraoral conversion to the cervical

\section{DISCUSSION}

A frog, this name was assigned long ago for liquid and encysted tumors of the floor of the mouth, this definition offers the advantage of not prejudging the nature of the affection, and we prefer it to that which took the frog like a dilation of the conduits or salivary glands [1].

It is a retentional cyst that develops on the floor of the mouth from the sublingual gland, salivary glands accessory to the floor of the mouth or the submandibular gland; It classically presents itself as a soft sub mucosal swelling in the oral floor. The term of frog comes from the resemblance between this cyst and the belly of a frog $[1,2]$.

A diving frog extends into the submandibular triangle of the neck across a defect in the mylohyoid muscle, or less frequently, passing behind the posterior edge of the muscle.A frog can also extend later in the parapharyngeal space [1].
It results in a rounded tumor, usually unilateral, painless, recurrent, translucent or bluish, from 3 to 10 millimeters in diameter. Sometimes it can reach 2 to 3 centimeters in diameter [1].

Like the mucoid cyst, the wall is thin, fragile and frequently ruptures. The cavity empties, but the cyst quickly re-forms. It occurs in children (or even newborns) as well as in adults and rests on the mylohyoid muscle, between the tongue and the alveolar bulwark. The salivary collection is often very large and extends into the lateral buccal floor, sometimes in the submandibular compartment, in an hourglass (bilobed cyst), up to sometimes the neck. The diagnosis of frog is easy, however, confusion with certain vascular malformations, or hemangioma may be possible. The bilobed forms are to be distinguished from dermoid cysts, cysts of the thyroglossal tract, and high branchial cysts [3].

The use of imagery is essential to assess the extension of this cyst and guide therapy. A possible CT scan could confirm the diagnosis. It is a uniloculated cyst with sometimes visible debris on ultrasound. The homogeneous and very high MRI signal in T2 is constant. Depending on the protein content of the secretion, the signal in $\mathrm{T} 1$ is hypo-intense or intermediate [3-5].

Exeresis of a diving frog starts with an incision horizontal is made in a fold, at minus $3 \mathrm{~cm}$ below the mandible or level of the hyoid bone, it extends forward from the anterior edge of the sterno muscle cleido mastoidien, through the skin, subcutaneous tissue and platysma. The veins common and anterior facial are spotted behind, dissected and ligated if necessary. The frog is identified in the anterior part of the submandibular triangle [1].

The anterior belly of the digastric is identified and reclined forward. The mylohyoid muscle is identified inside and in front of the belly digastric anterior. The surgeon may need to mobilize and dissect the submandibular gland for better access. Muscles encountered after excision of the submandibular gland, and dehiscence area of the mylohyoid muscle through which most often passes a plunging frog.The frog is mobilized by dissecting by cleavage along the surrounding muscles and behind the sub-mandilular gland. The frog usually goes through dehiscence in themylohyoid muscle or less frequently behind the mylohyoid muscle in the floor of the mouth. The surgeon completes so the oral excision associated with excision of the sublingual glands. The submandibular gland to be preserved, the state of the submandibular canal is checked to determine if it should be translocated. A mucosal defect in the floor is then closed with a absorbable suture and the neck is closed flat per plane on a suction drain [1]. For simple frogs, sometimes only marsupialization is possible [2]. 


\section{CONCLUSiON}

The diagnosis of frogs does not offer great diagnostic difficulties by their shape, their transparency and their softness, as well as the exploratory puncture with a fine needle makes it possible to rectify the diagnosis.

\section{REFERENCES}

1. The Open Access Atlas of Otolaryngology, Head \& Neck Operative Surgery by Johan Fagan (Editor) johannes.fagan@uct.ac.za is licensed under a Creative Commons Attribution - Non-Commercial 3.0 Unported License

2. Annales de Dermatologie et de Vénéréologie.2010; 137(12): 823-837.

3. Journal de Radiologie diagnostique et interventionnelle. 2013; 94(9): 921-926.

4. http://dx.doi.org/10.1016/j.crad.2007.12.007

5. Kurabayashi T, Ida M, Yasumoto $M$ and al. MRI of ranulas Neuroradiology. 2000; 42(12): 917-922. 